\title{
An Atypical Peripheral Nerve Sheath Tumour with Pseudoglandular Architecture in a Dog
}

\author{
Christelle VOLMER ${ }^{1) *}$, Laura CAPLIER ${ }^{1)}$, Edouard REYES-GOMEZ ${ }^{1)}$, Hélène HUET $^{1)}$, Roger A. OWEN ${ }^{2)}$ and \\ Jean-Jacques FONTAINE ${ }^{1)}$ \\ ${ }^{1)}$ Department of Pathology, National Veterinary School of Alfort, Maisons-Alfort Cedex and ${ }^{2)}$ Department of Safety Assessment, Merck \\ Sharp \& Dhome-Chibret Laboratories, Riom, France
}

(Received 13 August 2009/Accepted 18 October 2009/Published online in J-STAGE 27 November 2009)

ABSTRACT. This case describes a subcutaneous soft tissue tumour in a German Shepherd dog. Histologically, the lesion was characterized by proliferating ovoid cells, loosely arranged in a collagenous to myxoid stroma, and by numerous pseudoglandular structures lined by neoplastic cells. Immunohistochemically, neoplastic cells were labelled with vimentin, glial fibrillary acidic protein and S100 antibodies, but not with cytokeratin, desmin and smooth muscle actin antibodies. Ultrastructurally, neoplastic cells were characterized by numerous mitochondria surrounded by endoplasmic reticulum and contained few secondary lysosomes. This tumour was diagnosed as a subcutaneous peripheral nerve sheath tumour (PNST) with pseudoglandular architecture. This case illustrates the morphological diversity of PNST and provides new insight into the differential diagnosis of cutaneous tumours of similar morphology in the dog.

KEY WORDS: canine, peripheral nerve sheath tumours, pseudoglandular structures.

J. Vet. Med. Sci. 72(2): 249-251, 2010

Tumours arising from peripheral nerve sheaths are uncommonly encountered in dogs and are predominantly found in the nervous plexuses and less frequently in subcutaneous tissue [7]. These tumours can arise either from Schwann cells, perineural fibroblasts or both [5, 7]. Histologically, they are almost indistinguishable. In humans, benign tumours arising from Schwann cells can display Antoni type A or B pattern and Verocay bodies, and are known as Schwannomas. Malignant forms, classified as Malignant Peripheral Nerve Sheath Tumours, rarely present these patterns and may be difficult to diagnose without complementary exams [2]. In veterinary medicine, as these cellular configurations are infrequently observed, these tumours are classified as Peripheral Nerve Sheath Tumour (PNST), regardless of their precise cellular origin [5, 7]. Distinctive histomorphologic features are observed in some canine PNST like epithelioid variant, melanotic schwannomas, PNST with glandular differentiation, PNST with divergent mesenchymal differentiation and PNST with eosinophilic cytoplasmic globules $[3,5,6,8,10,11]$. Here, we report a PNST with a unique pseudoglandular architecture. By combining immunohistochemical and ultrastructural analyses, we identified this tumour as being of peripheral nerve sheath origin.

A 14-year-old female German Shepherd dog was presented to the National Veterinary School of Alfort (France) for an evaluation of a subcutaneous mass located to the right flank. The mass had a soft consistency, was not clearly demarcated and measured approximately $10 \mathrm{~cm}$ in diameter. A wide surgical resection was performed. Tissue-specimen

\footnotetext{
* Correspondence to: Volmer, C., Department of Pathology, National Veterinary School of Alfort, 7 avenue du Général de Gaulle, 94704 Maisons-Alfort Cedex, France.

e-mail: c.volmer@hotmail.fr
}

was fixed in $10 \%$ neutral-buffered formalin, routinely processed, sectioned at $4 \mu \mathrm{m}$-thickness, stained with haematoxylin, eosin and saffron (HES). Immunohistochemical evaluation was carried out on paraffin sections with the following primary antibodies: monoclonal mouse anti-vimentin (1:100, clone V9, Dako, DenmarkA/S, Golstrup, Denmark), monoclonal mouse anti-cytokeratin (1:500, clone AE1/AE3, Dako), monoclonal mouse anti-Glial Fibrillary Acidic Protein (GFAP, 1:100, clone 6F2, Dako), polyclonal rabbit anti-S100 (1:600, clone S100, Dako), monoclonal mouse anti-Desmin (1:50, clone D33, Dako), monoclonal mouse anti-Smooth Muscle Actin (SMA, 1:100, clone 1A4, Dako). Primary antibodies were revealed with a detection kit which comprised biotinylated secondary goat anti-mouse and goat anti-rabbit antibodies, a streptavidin horseradish peroxidase conjugate and diaminobenzidine chromogen (Ventana DAB detection kit, Ventana Medical Systems, Inc., Illkirch, France). Omission of primary antibody as negative control and positive control using reference tissues were included in each protocol. For ultrastructural evaluation, formalin-fixed specimen was refixed in a $4 \%$ formaldehyde/ $1 \%$ glutaraldehyde solution, processed routinely to epoxy-embedded tissue sections and observed with a Morgagni 268D transmission electron microscope (FEI company, Eindhoven, The Netherlands).

Gross examination of fixed specimen revealed a soft tissue, with many cystic areas containing a clear gelatinous fluid. Histological examination showed a subcutaneous, non encapsulated and poorly circumscribed tumour. The lesion consisted of a moderate cellular proliferation of noncohesive cells loosely arranged in a collagenous to myxoid stroma. Numerous clear spaces were seen, especially in areas of lower cellularity, and matched those observed on gross examination (Fig. 1). Their size ranged from approx- 
imately $100 \mu \mathrm{m}$ up to a few centimetres and sometimes contained a mucinous material or erythrocytes. These spaces were identified as pseudoglandular structures: they were lined by a pseudo-epithelium of variable thickness formed by neoplastic cells identical to those of the core of the tumour (Fig. 2). Cells were mostly ovoid, rarely fusiform, measured about $15 \mu \mathrm{m}$ in diameter and had an intermediate nuclear-cytoplasmic ratio. The cytoplasm was clear to acidophilic and contained discrete granulations that were more prominent in cells lining pseudoglands. The nucleus was central to paracentral, ovoid, euchromatic and nucleolated. Atypia was moderate (inconsistent prominent nucleoli and pleomorphic nuclei) and mitosis were rare $(<1$ per 10 high power field, $\times 400)$. Numerous capillaries had hyalinized walls (Fig. 2). Immunohistochemically, the cytoplasm of all neoplastic cells, including cells around pseudoglandular structures, were diffusely and strongly immunoreactive for vimentin, GFAP and S100 (Fig. 3A and 3B). No immunoreactivity was found for cytokeratin, desmin and SMA antigens. Ultrastructural analysis revealed round to ovoid cells with short cytoplasmic projections, surrounded by fibrillary material and few collagen fibrils. These cells contained abundant intermediate filaments and scattered cytoplasmic organelles (Fig. 4). These organelles were mostly mitochondria, surrounded by rough endoplasmic reticulum (RER) and few secondary lysosomes. These scattered organelles probably accounted for the fine cytoplasmic granulosity (Fig. 4). Pseudoglandular areas were not surrounded by a basement membrane. Based on the histologic, immunohistochemical and ultrastructural findings we conclude to a PNST.

The pseudoglandular architecture is a prominent feature of this tumour. These pseudoglandular structures could lead to some confusion with glandular structures occasionally observed in synovial sarcoma and PNST with glandular differentiation or with pseudoangiomatous formations in spindle cell lipoma $[4,11]$. However, glandular components are cytokeratin positive in the case of synovial sarcoma and in PNST with glandular differentiation. In the case of spindle cell lipoma, pseudoangiomatous pattern is organized around dilated vascular channels and is admixed with adipose tissue. In the present case, there was neither cytokeratin labelling nor adipose tissue.

Moreover, the lesion was characterized by a round to fusiform cell proliferation embedded in a myxoid stroma. Differential diagnosis of soft tissue tumour with myxoid stroma could include fibrosarcoma, hemangiopericytoma, myxosarcoma, embryonal rhabdomyosarcoma and myxoid liposarcoma. All of these tumours including PNST are vimentin-positive and can also express S100 antigen with the exception of fibrosarcoma and myxosarcoma $[1,4]$. As PNST, embryonal rhabdomyosarcoma has been reported to occasionally express GFAP [1]. Yet, the absence of desmin expression and sarcomere-like structures excluded a muscular origin. The lack of SMA labelling further excluded hemangiopericytoma. The absence of cytoplasmic lipid vacuoles, both histologically and ultrastructurally, ruled out
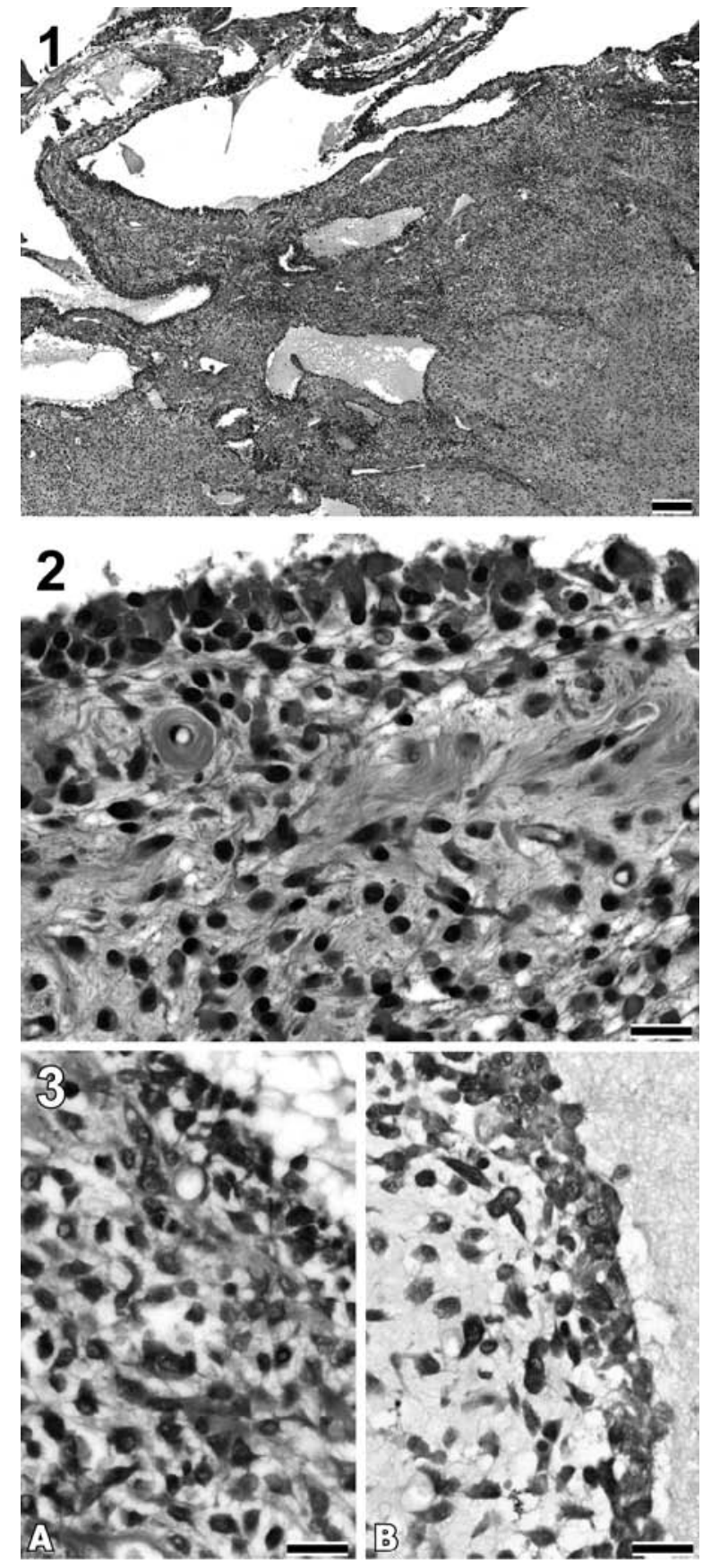

Fig. 1. Tumoral proliferation of non-cohesive cells admixed with a collagenous and myxoid stroma, containing numerous pseudoglandular structures filled with blood or mucinous material, HES. Bar $=200 \mu \mathrm{m}$.

Fig. 2. Neoplastic cells are mostly round-shaped and have a clear to acidophilic cytoplasm with discrete granulations, HES. Bar $=20 \mu \mathrm{m}$.

Fig. 3. A. Cytoplasm of neoplastic cells is diffusely and strongly positive for GFAP, immunoperoxidase staining, hematoxylin counterstain. Bar $=20 \mu \mathrm{m}$. B. Cytoplasm of neoplastic cells is diffusely and strongly positive for $\mathrm{S} 100$, immunoperoxidase staining, hematoxylin counterstain. $\mathrm{Bar}=20 \mu \mathrm{m}$. 


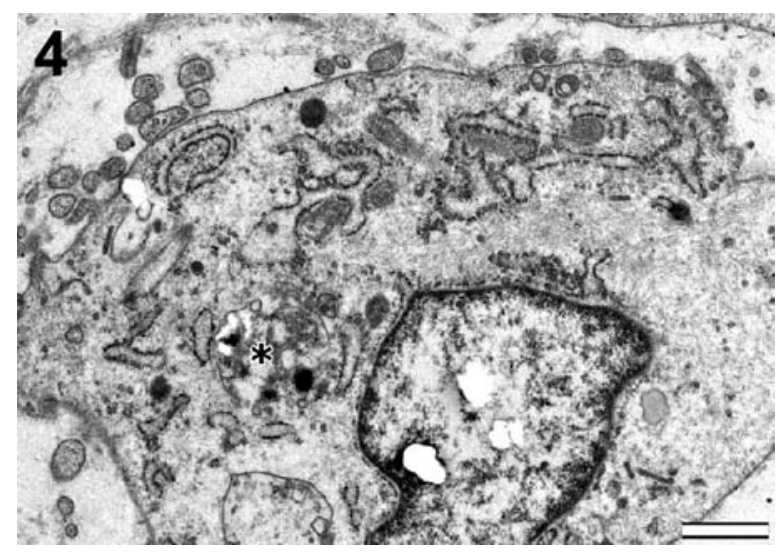

Fig. 4. Ovoid cell with short cytoplasmic projections, surrounded by fibrillary material. Cytoplasm contains numerous mitochondria surrounded by RER and few secondary lysosomes (asterisk), TEM. Bar $=1 \mu \mathrm{m}$.

an adipocytic origin [4].

Pseudoglandular schwannomas have been reported in humans and rats $[9,12]$. In those schwannomas, pseudoglandular elements are described as clear spaces delineated by pseudocolumnar to flattened cells laying on a condensed stroma. In the human study, most pseudoglandular elements are surrounded by Antoni type B loose tissue. These pseudoglandular elements are thought to arise from degenerative processes that could lead to cyst formation. According to the authors, the loose matrix of Antoni B area facilitates cyst expansion, which is followed by subsequent peripheral stroma and cell condensation forming a pseudoglandular wall [9]. Histomorphological findings of this canine tumour are thus reminiscent of the human and rodent cases. Nevertheless, this neoplasm was slightly different ultrastructurally in that it lacked a continuous basal lamina, which typically surrounds Schwann cells, and was only displaying some fibrillar material around few cells. This aspect might have been accentuated by formalin fixation in the present case. Although a Schwann cell origin was suspected in this tumour, the lack of a continuous basal membrane impedes a definitive diagnosis of Schwannoma. Yet, remnants of basal lamina around neoplastic cells are sometimes recorded in some human schwannomas and fragmented basal laminas are also noticed in cystic areas in human pseudoglandular schwannomas [2,9].

Even though this tumour was infiltrative it seemed to be of low grade of malignancy since after one year of follow-up there was neither evidence of recurrence nor metastasis.
ACKNOWLEDGMENT(S). The authors thank C. Grauliere, Merck Sharp \& Dhome-Chibret Laboratories (Riom, France), for helping with tissue processing for the electron microscopy.

\section{REFERENCES}

1. Chijiwa, K., Uchida, K. and Tateyama, S. 2004. Immunohistochemical evaluation of canine peripheral nerve sheath tumors and other soft tissue sarcomas. Vet. Pathol. 41: 307-318.

2. Erlandson, R. A. 1994. Diagnostic Transmission Electron Microscopy of Tumors with Clinicopathological, Immunohistochemical and Cytogenetic Correlations, Raven Press, New York.

3. Garcia, P., Sanchez, B., Sanchez, M. A., Gonzalez, M., Rollan, E. and Flores, J. M. 2004. Epithelioid malignant peripheral nerve sheath tumour in a dog. J. Comp. Pathol. 131: 87-91.

4. Gross, T. L., Ihrke, P. J., Walder, E. J. and Affolter, V. K. 2005. Part II: Mesenchymal tumors and other tumors. pp. 722 796. In: Skin Diseases of the Dog and Cat: Clinical and HistoPathologic Diagnosis, 2nd ed., vol. II, Blackwell Science, Ames.

5. Hendrick, M. J., Mhaffey, E. A., Moore, F. M., Vos, J. H. and Walder, E. J. 1998. Histological classification of mesenchymal tumors of skin and soft tissues of domestic animals. pp. 1-64. In: The WHO International Histological Classification of Tumors in Domestic Animal, vol. II, Armed Forced Institute of Pathology, Washington DC.

6. Kim, D. Y., Cho, D. Y., Kim, D. Y., Lee, J. and Taylor, H. W. 2003. Malignant peripheral nerve sheath tumor with divergent mesenchymal differentiations in a dog. J. Vet. Diagn. Invest. 15: 174-178.

7. Koestner, A. and Higgins, R. J. 2002. Tumors of the nervous system. pp. 731-735. In: Tumors in Domestic Animals, 4th ed. (Meuten D. J. ed.), Blackwell Science, Ames.

8. Kuwamura, M., Yamate, J., Kotani, T., Takeuchi, T. and Sakuma, S. 1998. Canine peripheral nerve sheath tumor with eosinophilic cytoplasmic globules. Vet. Pathol. 35: 223-226.

9. Laber-Laird, K. E., Jokinen, M. P. and Jerome, C. P. 1988. Naturally occurring schwannoma in a Fischer 344 rat. Vet. Pathol. 25: 320-322.

10. Patnaik, A. K., Erlandson, R. A. and Lieberman, P. H. 1984. Canine malignant melanotic schwannomas: a light and electron microscopic study of two cases. Vet. Pathol. 21: 483-438.

11. Patnaik, A. K., Zachos, T. A., Sams, A. E. and Aitken, M. L. 2002. Malignant nerve-sheath tumor with divergent and glandular differentiation in a dog: a case report. Vet. Pathol. 39: 406-410.

12. Robinson, C. A., Curry, B. and Rewcastle, N. B. 2005. Pseudoglandular elements in schwannomas. Arch. Pathol. Lab. Med. 129: 1106-1112. 